\title{
GUTs and Strings
}

\section{William A. Ponce}

Departamento de Física, Universidad de Antioquia, A.A. 1226, Medellín, Colombia

Abstract: We discuss the connection between string theories and Grand Unified Theories (GUTs), with particular emphasis on the list of available affine levels.

\section{KEYwORDS: Tunified theories, stringsi}

\section{Introduction}

Styring theory is the only candidate we have so far for a unification of all the fundamental interactions including gravity. At low energies one looks at the four dimensional field theory which results from string theory defined at the Planck scale. When one-loop effects are included in the perturbative heterotic string [1] unification of the gauge couplings at a scale $M_{s t r .} \sim$ $3.6 \times 10^{17} \mathrm{GeV}$.

Unification of coupling constants is a necessary phenomenon in string theory. Specifically, at tree level, the gauge couplings $\alpha_{i}=g_{i}^{2} / 4 \pi$, ( $i=1,2,3$, for the groups of the Standard Model $(\mathrm{SM})$ factors $U(1)_{Y}, \quad S U(2)_{L}$, and $S U(3)_{c}$ respectively) are related at the string scale by [i?

$$
\kappa_{3} \alpha_{3}=\kappa_{2} \alpha_{2}=\kappa_{1} \alpha_{1},
$$

where $\kappa_{i}, i=1,2,3$ are the affine levels, or KacMoody levels, at which the group factor $U(1)_{Y}$, $S U(2)_{L}$, and $S U(3)_{c}$ is realized in the four-dimensional string.

To calculate the Kac-Moody levels, the starting point is the ten-dimensional heterotic string with gauge group $S O(32)$ or $E_{8} \otimes E_{8}$ corresponding to an affine Lie algebra at level $\kappa=1$. A standard compactification [3in] leads to a four dimensional model with gauge group formed by a product of non-abelian gauge groups $G_{i}$ realized at levels $\kappa_{i}=1$, times $U(1)$ factors. Building string theories with non-abelian algebras at higher levels $(\kappa=2,3, \ldots)$ is considerable more difficult than at level one, and new methods for compactification must be developed [i]. Now, the affine levels for abelian $U(1)$ factors can not be determined from algebraic procedures and their values may be considered as free parameters in the four dimensional string $[\bar{i}$.

Then, the compactification of the heterotic string to the four dimensional $G_{S M} \equiv S U(3)_{c} \otimes$ $S U(2)_{L} \otimes U(1)_{Y}$ could be achieved at $M_{s t r .}$, with $\kappa_{2}, \kappa_{3}=1,2, \ldots n$, an integer number, and $\kappa_{1}$ a normalization free coefficient $\left(\kappa_{1}>1\right.$ in order for the $e_{R}$ to be in the massless spectrum of the four dimensional string $[\overline{6}|\overline{\mid}|$ ). The compactification to a four dimensional simple gauge group $G\left(=S U(5), S O(10), E_{6}\right.$, etc.) has also been partially studied in the literature, with upper values for the integer $\kappa$ levels calculated [i]| Also, strings with $S U(5) \subset S U(5) \otimes S U(5)$ and $S O(10) \subset S O(10) \otimes S O(10)$ at levels $\kappa_{2}=\kappa_{3}=2$ have been presented in Ref. [i $4_{1}^{\overline{1}}$.

The values attained by level $\kappa_{i}$ play a fundamental role in string theories, because they fix at the string scale the electroweak mixing angle $\sin \theta_{W}$. Besides, they impose limits on possible representations allowed at low energies [iי4], and determine the conformal spin of the currents $J$ which are forced to be in the spectrum because of charge quantization $[\overline{6} \mid$. So, theories with different $\kappa_{i}$ values must have quite different physical implications.

Today it is believed that $M_{\text {str. }}$ could be not the perturbative value $3.6 \times 10^{17} \mathrm{GeV}$, but a smaller one (maybe as small as $1 \mathrm{TeV}$ ) [i. from the non perturbative effects of the string. This matter has not been settled yet, and it is not crucial for the analysis which follows. 


\section{Unified Theories}

In a particular GUT model, the unification of the three SM gauge couplings is properly achieved if they meet together into a common value $\alpha=$ $g^{2} / 4 \pi$ at a certain energy scale $M$, where $g$ is the gauge coupling constant of the unifying group $G$. However, since $G \supset G_{S M}$, the normalization of the generators corresponding to the subgroups $U(1)_{Y}, S U(2)_{L}$, and $S U(3)_{c}$ is in general different for each particular group $G$, and therefore the SM coupling constants $\alpha_{i}$ differ at the unification scale from $\alpha$ by numerical factors $c_{i}\left(\alpha_{i}=c_{i} \alpha\right)$. As a matter of fact, if $\alpha_{i}$ is the coupling constant of $G_{i}$, a simple group embedded into $G$, then

$$
c_{i} \equiv \frac{\alpha_{i}}{\alpha}=\frac{\operatorname{Tr} \cdot T^{2}}{\operatorname{Tr} \cdot T_{i}^{2}}
$$

where $T$ is a generator of the subgroup $G_{i}$ properly normalized over a representation $R$ of $G$, and $T_{i}$ is the same generator but normalized over the representations of $G_{i}$ embedded into $R$ (the traces run over complete representations); so, if just one standard doublet of $S U(2)_{L}$ is contained in the fundamental representation of $G$ (plus any number of $S U(2)_{L}$ singlets), then $c_{2}=1$ (as in $S U(5)[9.9$ for example). In this way we proof that for $i=2,3, c_{i}^{-1}$ is an integer number.

The constants $c_{i}$ are thus pure rational numbers satisfying $c_{1}>0$, and $0<c_{2(3)} \leq 1$. They are fixed once we fix the unifying gauge structure, and from pure algebraic arguments we must have at the GUT scale

$$
c_{3}^{-1} \alpha_{3}=c_{2}^{-1} \alpha_{2}=c_{1}^{-1} \alpha_{1} .
$$

In Table 1 we present the $c_{i} i=1 ; 2 ; 3$ values for most of the GUT groups in the literature; they are calculated using Eq.(2..1 $)$. The canonical entry is associated with the following

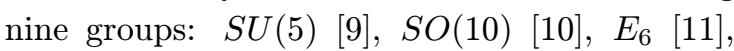
$[S U(3)]^{3} \times Z_{3} \quad[12], S U(15)$ [1]

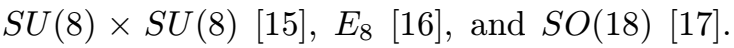
The model $[\mathrm{SU}(3)]^{4} \times Z_{4}$ is taken from Reference [1 $18 \overline{1}$ ], $S U(5) \otimes S U(5)$ from from [201], $[S U(6)]^{3} \times Z_{3}$ from [21, ${ }_{1}^{2},[S U(6)]^{4} \times Z_{4}$ from $\left[22 i, E_{7}\right.$ from $\left[23^{n}\right],[S U(4)]^{3} \times Z_{3}$ from $\left[2{ }^{2}\right]$, and $[S U(2 F)]^{4} \times Z_{4}$ (the Pati-Salam models for $F$ families) from $[24]$.
In the canonical entry we have normalized the $c_{i}$ values for some groups to the $\mathrm{SU}(5)$ numbers; for example, the actual values for $\mathrm{SO}(10)$ are $\left\{c_{1}^{-1} ; c_{2}^{-1} ; c_{3}^{-1}\right\}=\{10 / 3 ; 2 ; 2\}=2\{5 / 3 ; 1 ; 1\}$, and for SU(16) are $\left\{c_{1}^{-1} ; c_{2}^{-1} ; c_{3}^{-1}\right\}=\{20 / 3 ; 4 ; 4\}=$ $4\{5 / 3 ; 1 ; 1\}$. This normalization makes sense because the common factor can be absorbed in the GUT coupling constant $\alpha$; besides, physical quantities such as $\sin ^{2} \theta_{W}, M_{G U T}$, etc., depend only on ratios of the $c_{i}$ values.

$c_{3}^{-1}$ can take only the values $1,2,3,4$ for one family groups, or higher integer values for family groups. $c_{3}^{-1}=1$ when it is $S U(3)_{c}$ which is embedded in the GUT group $G ; c_{3}^{-1}=2$ when it is the chiral color [25] $S U(3)_{c L} \times S U(3)_{c R}$ which is embedded in $G$, etc. For example $c_{3}^{-1}=4$ in $\mathrm{SU}(16)$ due to the fact that the color group in $\mathrm{SU}(16)$ is $S U(3)_{c u R} \times S U(3)_{c d R} \times S U(3)_{c u L} \times$ $S U(3)_{c d L}$.

For some family groups $c_{2}^{-1}$ take the values $1,2, \ldots F$ for $1,2, \ldots F$ families. Indeed, the $c_{i}$ values for the $F$ family Pati-Salam models [24"] $[S U(2 F)]^{4} \times Z_{4}$ are $\left\{c_{1}^{-1} ; c_{2}^{-1} ; c_{3}^{-1}\right\}=\{(9 F-$ $8) / 3 ; F ; 2\}$; and for $[S U(2 F)]^{3} \times Z_{3}=S U(2 F)_{L} \otimes$ $S U(2 F)_{c} \otimes S U(2 F)_{R} \times Z_{3}$ (the $2 F$ color vec-

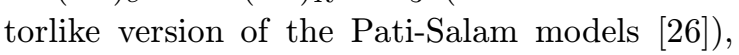
$\left\{c_{1}^{-1} ; c_{2}^{-1} ; c_{3}^{-1}\right\}=\{(6 F-4) / 3 ; F ; 1\}$.

In general, $c_{2(3)}^{-1}=1,2, \ldots f$, where $f$ is the number of fundamental representations of $S U(2)_{L}$ $\left(S U(3)_{c}\right)$ contained in the fundamental representation of the GUT group. For example, $c_{2}^{-1}=$ 4 in $S U(16)$ because the 16 representation of $S U(16)$ contains four $S U(2)_{L}$ doublets; three for $(u, d)_{L}$ and one for $\left(\nu_{e}, e\right)_{L}$.

The group $[S U(4)]^{3} \times Z_{3}$ in Table 1 is not the vector-like color version of the two family PatiSalam model, but it is the one family model introduced in Ref. [20]. The group $[S U(6)]^{4} \times Z_{4}$ in the Table could be the three family Pati-Salam model [24], or either the version of such a model without mirror fermions introduced in Ref. [22]. $E_{7}$ is defined in Ref[[23].

Notice that the values for $c_{1}^{-1}$ are integer multiple of $1 / 3$ for all the groups in the table, which is due to the condition for having only standard electric charges in the representations of the particular group used as a GUT. Such con- 
dition reads

$$
c_{1}^{-1}+c_{2}^{-1}+\frac{4}{3} c_{3}^{-1}=0 \quad \bmod .4
$$

which is satisfied by all entries in the table (in some entries the real values must be used instead of the normalized ones).

\section{String-GUTs}

The logarithmic running through the "desert" of the fundamental coupling constant is governed by the following renormalization group equations:

$$
\alpha_{i}^{-1}(\mu)=\eta_{i} \alpha^{-1}-\frac{b_{i}}{2 \pi} \ln \left(\frac{M}{\mu}\right)+\Delta_{i}
$$

where $b_{i}$ are the one-loop beta functions, $M$ the unification scale and $\Delta_{i}$ the threshold and other corrections.

GUTs (and SUSY-GUTs) were invented [9]" before strings, and they may exist by themselves as independent physical entities. For the several GUT models $\eta_{i}=c_{i}^{-1}$ in Eq. (3.1), $M=$ $M_{G U T}$ is the GUT scale, and $\alpha=g^{2} / 4 \pi$, with $g$ the coupling constant of the GUT group. However, it is a well known result that the logarithmic running through the desert of the three gauge couplings $c_{i} \alpha_{i}^{-1}$ for the canonical values $\left\{c_{1}, c_{2}, c_{3}\right\}=\left\{\frac{3}{5}, 1,1\right\}$, do merge together into a single point, only when the SUSY partners of the SM elementary particles are included in the renormalization group equations at a mass scale $M_{\text {susy }} \sim 1 \mathrm{TeV}\left[{ }^{2} \overline{7}_{1}\right]$. This amazing result is not upset when higher order contributions are taken into account [2 $\left.{ }^{-} \overline{8}^{\prime}\right]$, and it provides the unification scale $M_{G U T} \sim 2 \times 10^{16} \mathrm{GeV}$.

Now, strings may exist without GUTs. If this is the case, then the string must compactify to four dimensions to the SM gauge structure $G_{S M}$ at a mass scale $M_{\text {string }}$. The fundamental coupling constants still run according to Eq. $(\overrightarrow{3} .1)$, where now $M=M_{\text {string }}, \eta_{i}=\kappa_{i}$ and $\alpha=g_{\text {string }}^{2} / 4 \pi$. When we solve Eqs.(3.1) using the one loop SUSY beta functions $\left(b_{1}, b_{2}, b_{3}\right)=$ $(-11,-1,3)$, and the canonical Kac-Moody levels $\left\{\kappa_{1}, \kappa_{2}, \kappa_{3}\right\}=\left\{\frac{5}{3}, 1,1\right\}$, we get $M_{\text {str. }}=2 \times$ $10^{16} \mathrm{GeV}$, a factor of 20 smaller than its perturbative value. If we solve the equation for $M=M_{\text {str. }}=3.6 \times 10^{17} \mathrm{GeV}$, we get $\kappa_{1}=\frac{5}{4}$ [29']. This is the so call string-GUT problem.
But it may happen that string-GUTs are real objects. They exist if the string compactifies in four dimensions not to $G_{S M}$, but to a simple group $G$ that acts as a unified group. If this is the case, then $M=M_{G U T}=M_{s t r}$, and $\eta_{i}=c_{i}^{-1}=\kappa_{i}$, which become two necessary conditions for having a consistent string-GUT. In this kind of theories not only the entire unification of interactions is realized, but also the advantages of the GUT symmetry are available.

\section{Conclusions}

In this note most of the four dimensional string Kac-Moody levels which could be related to GUT theories are presented in Table 1, from where we may visualize the wide spectrum available for the values $\kappa_{i}=c_{i}^{-1}, i=1,2,3$.

So far, almost the entire literature on four dimensional strings has been focused on the canonical values $\kappa_{2}=\kappa_{3}=1, \kappa_{1}=5 / 3$, pointing towards a canonical string-GUT model, or to a string model without a relation to a particular GUT. But as it is known, there are serious problems with the models constructed so far. Just to mention a few we have: the string-GUT problem, the doublet-triplet problem [i $\left[\begin{array}{l}4 \\ 1\end{array}\right]$, the failure to produce a consistent low energy particle spectrum [i4], etc. It may be feasible that the construction of four dimensional string theories with non-canonical $\kappa_{i}$ values may cure some of the mentioned problems (in the model of Ref. [1 $18 \overline{1}$, $M_{G U T} \geq M_{s t r}$, and the doublet triplet problem is not present at tree level).

$\kappa_{i}, i=1,2,3$ values, different from the canonical ones, are in general related to the existence of non standard matter. That extra matter can have a mass at an intermediate scale, or either at the string-GUT scale.

\section{Acknowledgments}

This work was partially supported by Colciencias and BID in Colombia.

\section{References}

[1] V.Kaplunovsky, Nucl. Phys. B307, 145 (1988); ibid B382, 436 (1992). 


\begin{tabular}{||l|c|c|c||}
\hline Group & $c_{1}^{-1}$ & $c_{2}^{-1}$ & $c_{3}^{-1}$ \\
\hline \hline Canonical (9 groups) & $5 / 3$ & 1 & 1 \\
\hline$[\mathrm{SU}(3)]^{4} \times Z_{4}$ & $5 / 3$ & 1 & 2 \\
\hline $\mathrm{SU}(5) \otimes \mathrm{SU}(5), \mathrm{SO}(10) \otimes \mathrm{SO}(10)$ & $13 / 3$ & 1 & 2 \\
\hline$[S U(6)]^{3} \times Z_{3}$ & $14 / 3$ & 3 & 1 \\
\hline$[S U(6)]^{4} \times Z_{4}$ & $19 / 3$ & 3 & 2 \\
\hline$E_{7}$ & $2 / 3$ & 2 & 1 \\
\hline$[S U(4)]^{3} \times Z_{3}$ & $11 / 3$ & 1 & 1 \\
\hline$[S U(2 F)]^{4} \times Z_{4}$ & $(9 F-8) / 3$ & $F$ & 2 \\
\hline$[S U(2 F)]^{3} \times Z_{4}$ & $(6 F-4) / 3$ & $F$ & 1 \\
\hline
\end{tabular}

Table 1: $c_{1}, c_{2}$ and $c_{3}$ values for most of the GUT models in the literature. $F=1,2, \ldots$ stands for the number of families in that particular model. The 9 "canonical" groups are presented in the main text.

[2] P.Ginsparg, Phys. Lett. B197, 139 (1987).

[3] L.Dixon, J.Harvey, C.Vafa, and E.Witten, Nucl. Phys. B261, 620 (1985); B274, 285 (1986); L.E.Ibañez, J.Mas, H.P.Nilles, and F.Quevedo, Nucl. Phys. B301, 137 (1988).

[4] A.Font, L.E.Ibañez, and F.Quevedo, Nucl. Phys. B345, 389 (1990); G.Aldazabal, A.Font, L.E.Ibañez, and A.M.Uranga, Nucl. Phys. B452, 3 (1995); B465, 34 (1996).

[5] L.E.Ibañez, Phys. Lett. B303, 55 (1993); B318,73 (1993).

[6] A.N.Schellekens, Phys. Lett. B237, 363 (1990).

[7] J.Ellis, J.L.López, and D.V.Nanopoulos, Phys. Lett. B245, 375 (1990).

[8] I. Antoniadis, Phys. Lett. B246, 377 (1990); I. Antoniadis, K. Benakli and M. Quirós, Phys. Lett. B331, 313 (1994).

[9] H.Georgi and S.L.Glashow, Phys. Rev. Lett. 32, 438 (1974); H.Georgi, H.R.Quinn, and S. Weinberg, Phys. Rev. Lett. 33, 451 (1974).

[10] H.Georgi, in Particles and Fields-1974, edited by C.E.Carlson (American Institute of Physics, N.Y. 1975), p. 575; H. Frietzsch and P. Minkowsky, Ann. Phys. 93, 193 (1975).

[11] F.Gürsey, P.Ramond and P.Sikivie, Phys. Lett. B60, 177 (1975)

[12] A. de Rújula, H.Georgi, and S. Glashow, in Fifth Workshop on Grand Unification, edited by K.Kang, H.Fried, and P.Frampton (World Scientific, Singapore, 1984), p. 88.

[13] S.L.Adler, Phys. Lett. B225, 143 (1989); P.H.Frampton and B.H.Lee, Phys. Rev. Lett. 64, 619 (1990).
[14] J.C.Pati, A.Salam, and J.Strathdee, Phys. Lett. B108, 121 (1982); N.G.Deshpande, E.Keith, and P.B.Pal, Phys. Rev. D47, 2893 (1993).

[15] N.G.Deshpande and P.Mannheim, Phys. Lett B94, 355 (1980).

[16] I.Bars and M.Gunaydin, Phys. Rev. Lett. 45, 859 (1980); S.M.Barr, Phys. Rev. D37, 204 (1998).

[17] F.Wilczek and A.Zee, Phys. Rev. D25, 553 (1982); J.Bagger et al, Nucl. Phys. B258, 565 (1985).

[18] A.Pérez-Lorenzana and W.A.Ponce, Phys.Lett. B464, 77 (1999).

[19] A.Davidson and K.C.Wali, Phys. Rev. Lett. 58, 2623 (1987); R.N.Mohapatra, Phys. Lett B379, 115 (1996).

[20] P.Cho, Phys. Rev. D48, 5331 (1993).

[21] A.H-Galeana, R.Martínez, W.A.Ponce, and A.Zepeda, Phys. Rev. D44, 2166 (1991); W.A.Ponce and A.Zepeda, Phys. Rev. D48, 240 (1993).

[22] W.A.Ponce and A.Zepeda, Z. Physik C63, 339 (1994); A. Pérez-Lorenzana, W.A. Ponce and A. Zepeda, Rev. Mex. Fis. 45, 109 (1999).

[23] F.Gürsey and P.Sikivie, Phys. Rev. Lett. 36, 775 (1976); Phys. Rev. D16, 816 (1977).

[24] J.C.Pati and A.Salam, Phys. Rev. Lett. 31, 661 (1973); V.Elias and S.Rajpoot, Phys. Rev. D20, 2445 (1979).

[25] J.C.Pati and A.Salam, Nucl. Phys. B50, 76 (1979); P.H.Frampton and S.L.Glashow, Phys. Lett. B190, 157 (1987).

[26] W.A.Ponce and A. Pérez-Lorenzana, Europhysics Lett. 49(3), 296 (2000). 
[27] U.Amaldi, W.de Boer, and H.Furstenau, Phys. Lett. B260, 447 (1991); P.Langacker and M.Luo, Phys. Rev. D44, 817 (1991).

[28] P.Langacker and N.Polonsky, Phys. Rev. D47, 4028 (1993).

[29] A.Pérez-Lorenzana, W.A.Ponce, and A.Zepeda, Europhysics Lett. 39(2), 141 (1997); Mod. Phys. Lett. A13, 2153 (1998). 